\title{
Physics of the very early universe: what can we learn from cosmological observations?
}

\author{
Paolo Gondolo \\ Department of Physics \\ University of Utah \\ 115 S 1400 S Rm 201 \\ Salt Lake City, UT 84112-0830 \\ USA \\ Email: paolo@physics.utah.edu
}

\begin{abstract}
Cosmological observations are starting to probe the evolution of the Universe before nucleosynthesis. The observed fluctuations in the cosmic microwave background and in the distribution of matter can be traced back to their origin during inflation, and the inflaton potential has begun to be unraveled. A future probe of the first microseconds would be the detection of weakly-interacting massive particles as dark matter. Discovery of supersymmetric particles at odds with the standard cosmological lore may open an experimental window on the physics at the highest energies, perhaps as far as superstring theory. This presentation will overview two topics on the physics of the Universe before nucleosynthesis: (1) slow-roll, natural and chain inflation in the landscape, and (2) neutralino dark matter production from inflatons or moduli fields.
\end{abstract}

CMB and Physics of the Early Universe 20-22 April 2006

Ischia, Italy 


\section{Contents}

1. Slow roll, natural, and chain inflation 3

2. Supersymmetric dark matter from moduli fields $\quad 6$

3. Conclusions 10

The cosmological Big Bang model rests on three pillars: the large-scale isotropy and homogeneity of the Universe, the cosmic microwave background (CMB), and primordial nucleosynthesis (BBN). The first two pillars relate to the history of the Universe after its first $\sim 100,000$ years. The third pillar concerns the Universe between $\sim 1 \mathrm{sec}$ and $\sim 3 \mathrm{~min}$. What happens during the first second is still being explored.

A lot of interesting physics is conjectured to have happened before Big Bang nucleosynthesis. These are the most energetic times in the history of the world, and are thus connected to the physics of the highest energies and smallest scales. At very early times, inflation has quickly expanded the primordial Universe so that a small causally-connected homogeneous patch now encompasses the whole visible Universe. Inflation left the Universe in an extremely cold state, and the processes of reheating raised its temperature and entropy to the levels observed today and inferred at primordial nucleosynthesis. Sometime during the first second, the mechanism of baryogenesis created more matter than antimatter so that today only matter is observed in the Universe. New fundamental symmetries of physics, like supersymmetry, may have been manifest in the early hot periods. At the very early times, the quantum aspects of gravity may have been important, and superstring theory may offer guidance on the workings of the primeval stages.

Several probes are at our disposal, in principle or in practice, to uncover the history of the Universe before nucleosynthesis and test our theoretical ideas. Inflation determines the initial pattern of density variations that become the fluctuations in the cosmic microwave background and in the distribution of matter at large scales. Their detection in the last decade is giving us more and more information on the details of inflation. Other probes of the very early Universe are particles that were produced in those ancient times and survive until the present day, relics of the early Universe. For example, we should be immersed in a sea of primordial neutrinos analogous to the photons of the cosmic microwave background. Unfortunately, we currently lack feasible ideas on how to detect them. Elementary particle candidates for non-baryonic cold dark matter would also be typically produced before nucleosynthesis, in the first microsecond or earlier. Many experiments are currently searching for them.

This presentation focuses on two ideas that may connect the current or planned cosmological observations to fundamental physics at the highest energy scales, perhaps as far as superstring theory. The two topics are: inflation (slow roll, natural, and chain inflation in the landscape), and supersymmetric dark matter from inflatons or moduli fields. 


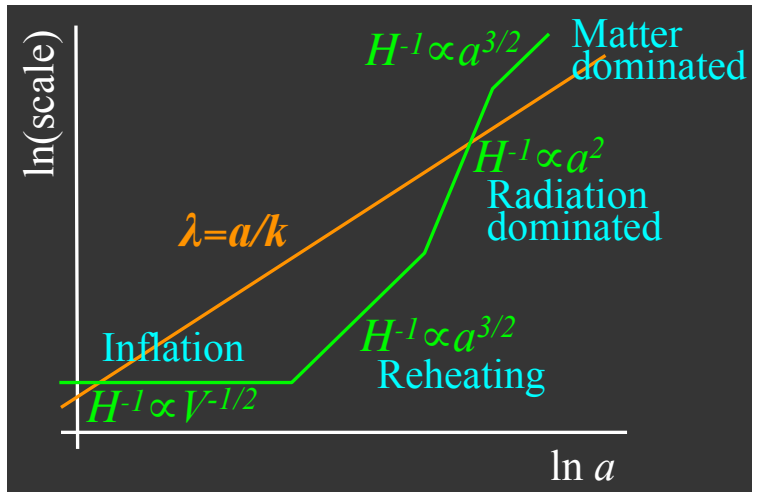

Figure 1: Schematic plot of the dependence of a generic wave-length $\lambda$ and of the Hubble radius $H^{-1}$ on the scale factor $a$. Here $k$ is the comoving wave-number.

\section{Slow roll, natural, and chain inflation}

Cosmological puzzles unresolved by the standard big bang model are (a) the smoothness (homogeneity and isotropy) at the largest scales, (b) the flatness of the three-dimensional geometry of space, (c) the oldness of the Universe when compared to its gravitational time scale, and (d) the absence of monopoles or other topological remnants from very early phase transitions. The idea of inflation was proposed to resolve these puzzles [1]. As a bonus, it has supplied a mechanism for the causal generation of the density perturbations required for galaxy formation.

The basic idea of inflation is to start with a region of space that is so small that causal processes had the time to homogenize it. The region is then stretched so rapidly that it becomes much bigger than the horizon distance, i.e. the distance light can cover since the beginning of the time. In this way, places that at the time of the CMB formation were separated by more than one horizon distance can share the same temperature since they originated from the same small region of space.

This idea is represented schematically in figure 1 . As the Universe expands, any wave-length $\lambda$ increases proportionally to the scale factor $a$, as $\lambda=a / k$. The proportionality constant is fixed by the comoving wave-number $k$. The Hubble radius $H^{-1}$, which is related to the size of the horizon, can increase faster or slower than $\lambda$. In fact, the Hubble parameter $H$ is related to the total energy density of the Universe $\rho$ through the Friedmann equation,

$$
H^{2}=\frac{8 \pi}{3 M_{\mathrm{Pl}}^{2}} \rho
$$

and its dependence on $a$ is controlled by the dominant form of energy. When non-relativistic matter is dominant, the energy density decreases as $a^{-3}$, and it follows that $H^{-1}$ increases as $a^{3 / 2}$. When relativistic radiation dominates, the energy density falls as $a^{-4}$, due to the decrease in frequency, and $H^{-1}$ grows as $a^{2}$. In both of these cases, the Hubble radius increases faster than any wavelength. During inflation, however, when the energy density is dominated by the potential energy density $V(\phi)$ of a scalar field $\phi$ (the inflaton), the Hubble radius is proportional to $\sqrt{V(\phi)}$, which can increase logarithmically slowly or remain constant for a long period of time. In this regime, a given wave-length can become larger than the horizon. For example, the wave-length depicted in figure 1 exits the horizon at early times (small $a$ ) and reenters it at late times (large $a$ ). 
A specific model of inflation is defined by the choice of inflaton potential $V(\phi)$, which may depend on many scalar fields, $\phi_{1}, \phi_{2}, \phi_{3}, \ldots$ The concept of single-field inflation covers both inflationary theories with a single scalar field (which could be fundamental, such as a modulus field in superstring theory, or macroscopic, such as an order parameter) and effective theories reduced to a single scalar by integration of other degrees of freedom. Examples of single-field inflaton potentials are:

$$
\begin{array}{llrl}
V(\phi) & =\Lambda^{4}(\phi / \mu)^{p} \quad \text { (chaotic inflation), } & V(\phi) & =\Lambda^{4} \exp ( \pm \phi / \mu) \quad \text { (power-law inflation), } \\
V(\phi) & =\Lambda^{4}\left[1+(\phi / \mu)^{p}\right] \quad \text { (hybrid inflation), } V(\phi)=\Lambda^{4}[1+\cos (\phi / \mu)] \quad \text { (natural inflation) }
\end{array}
$$

Here $\Lambda$ and $\mu$ are parameters with the dimension of mass, and $p$ is an arbitrary real number.

To ensure that $V(\phi)$, and thus $H^{-1}$, is constant for a long time, the potential is often chosen so that its derivatives are small. Quantitatively, one defines a set of so-called slow-roll parameters which are then constrained to be tiny. A common definition is

$$
\varepsilon(\phi) \equiv \frac{M_{\mathrm{Pl}}^{2}}{4 \pi}\left(\frac{H^{\prime}}{H}\right)^{2} \simeq \frac{M_{\mathrm{Pl}}^{2}}{16 \pi}\left(\frac{V^{\prime}}{V}\right)^{2} \ll 1, \quad \eta(\phi) \equiv \frac{M_{\mathrm{Pl}}^{2}}{4 \pi} \frac{H^{\prime \prime}}{H} \simeq \frac{M_{\mathrm{Pl}}^{2}}{8 \pi}\left[\frac{V^{\prime \prime}}{V}-\frac{1}{2}\left(\frac{V^{\prime}}{V}\right)^{2}\right] \ll 1,
$$

where a prime indicates a derivative with respect to $\phi$, and $H(\phi)$ is obtained through the Friedmann equation.

Even the restricted class of slow-roll single-field potentials still contains many models of inflation: new, chaotic, natural, power-law, modulus, D-term, F-term, etc. A useful classification scheme has been introduced in [2]. Potentials that vanish at the origin with a positive second derivative are called large-field models. Potentials that do not vanish at the origin are called either small-field models (if their second derivative at the origin is negative) or hybrid models (if it is positive). This classification conveniently translates into separate ranges of slow-roll parameters, namely $\eta<-\varepsilon$ (small-field), $-\varepsilon<\eta<\varepsilon$ (large-field), and $0<\varepsilon<\eta$ (hybrid). It also translates into separate regions in the observable parameters $n_{s}$ and $r$ defined below.

Inflationary models are examined observationally by means of the fluctuation spectra they predict in the $\mathrm{CMB}$ and the matter distribution. Commonly used parameters in this context are: the amplitude of the density perturbations $\delta \rho / \rho \propto V^{3 / 2} / V^{\prime}$, the spectral index of the density perturbations $n_{s} \simeq 1-4 \varepsilon+2 \eta$, with $\varepsilon \propto\left(V^{\prime} / V\right)^{2}$ and $\eta \propto V^{\prime \prime} / V$, the running spectral index $n_{s}(k)$, which contains higher derivatives of $V$, the amplitude of the gravitational waves generated during inflation, which is $\propto V$, and the spectral index of the gravitational waves $n_{T} \propto \varepsilon$. A complete measurement of these parameters would provide information on the functional form of the inflaton potential (and perhaps enable its full reconstruction, see [3] for a summary of the latter).

The calculation of the primordial scalar and tensor spectra from quantum fluctuations during slow-roll single-field inflation can be summarized by the following procedure (see $[4,5]$ for detailed derivations). For each comoving wave-number $k$, (1) solve the following equation for the value $\phi_{k}$ the field has when the wave-length $\lambda=a / k$ exits the horizon (leftmost crossing in figure 1):

$$
\frac{2 \sqrt{\pi}}{M_{\mathrm{Pl}}} \int_{\phi_{e}}^{\phi_{k}} \frac{\mathrm{d} \phi}{\sqrt{\varepsilon(\phi)}}=62-\ln \frac{k V_{e}^{1 / 3} 10^{16} \mathrm{GeV}}{a_{0} H_{0} V_{k}^{1 / 2} \rho_{\mathrm{RH}}^{1 / 12}}
$$


(this condition equates two expressions of the number of e-foldings $N_{k}=\ln a_{e}-\ln a_{k}$ between the horizon crossing at $a=a_{k}$ and the end of inflation at $a=a_{e}$ - here $\phi_{e}$ is the field at the end of inflation, $V_{e}=V\left(\phi_{e}\right), V_{k}=V\left(\phi_{k}\right), \rho_{\mathrm{RH}}$ is the radiation density at the end of reheating, and $a_{0}, H_{0}$ are the scale factor and the Hubble parameter at the end of the last matter dominated epoch). (2) Use the value of $\phi_{k}$ in the expressions for the spectra

$$
P_{s}(k)=\frac{H\left(\phi_{k}\right)^{2}}{4 \pi^{2} M_{\mathrm{Pl}}^{2} \varepsilon\left(\phi_{k}\right)}, \quad P_{T}(k)=\frac{H\left(\phi_{k}\right)^{2}}{4 \pi^{2} M_{\mathrm{Pl}}^{2}} .
$$

This procedure gives the complete $k$-dependence of the scalar and tensor power spectra $P_{S}(k)$ and $P_{T}(k)$. It is customary to introduce running spectral indices $n_{s}(k)$ and $n_{T}(k)$, defined by

$$
P_{S}(k) \propto k^{n_{s}(k)}, \quad P_{T}(k) \propto k^{n_{T}(k)} .
$$

In the slow-roll approximation, one obtains $n_{s}(k) \simeq 1-4 \varepsilon\left(\phi_{k}\right)+2 \eta\left(\phi_{k}\right)$ and $n_{T}(k) \simeq-2 \varepsilon\left(\phi_{k}\right)$. Moreover, the ratio $r(k)$ of the tensor-to-scalar amplitudes is approximately $r(k) \simeq 16 \varepsilon\left(\phi_{k}\right)$.

How to best represent the functions $n_{s}(k)$ and $n_{T}(k)$ in terms of few parameters is still under study. The WMAP collaboration uses an expansion of $n_{s}(k)$ in powers of $\ln k$ around a fiducial scale $k_{0}=0.002 \mathrm{Mpc}^{-1}[6]$. When keeping only the constant term, they are able to exclude $\lambda \phi^{4}$ inflation models with more than $\sim 58$ e-foldings at the $95 \%$ C.L. The constraints change noticeably when the expansion is continued up to the first derivative. Furthermore, the constraints are considerably altered if the expansion of $n_{s}(k)$ in powers of $\ln k$ is replaced by an expansion of $H(\phi)$ in powers of $\phi$, both to first order [7]. This parametrization dependence of the constraints will have to be understood before the implications of the data can be fully established.

The rest of this section discusses two inflation models that may naturally be present in superstring theory: natural inflation and chain inflation.

Natural inflation $[8,9]$ starts from the realization that a very flat inflaton potential is unnatural in particle physics, for example $V=\lambda \phi^{4}$ requires $\lambda<10^{-12}$. Natural inflation uses axion-like

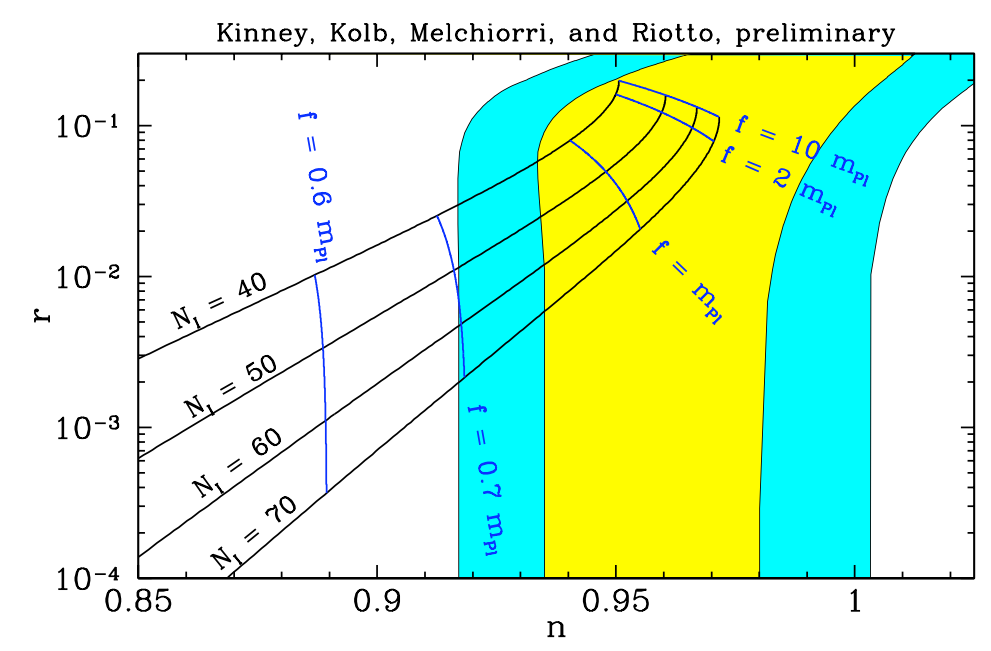

Figure 2: WMAP three year constraints on natural inflation (from [10]). The yellow (light grey) region is excluded at 68\% C.L., the blue (dark grey) region at 95\% C.L. The curves show the predictions of natural inflation at various values of the number of e-foldings $N_{I}$ and the scale $f$. 
potentials $V=\Lambda^{4}[1+\cos (\phi / f)]$ to protect flatness without small dimensionless parameters. The scale $\Lambda$, of order the grand-unification scale $\left(\Lambda \sim M_{\mathrm{GUT}}\right)$, sets the amplitude of the scalar perturbations, while a separate scale $f \sim M_{\mathrm{Pl}}$ guarantees enough inflation, i.e. an adequate number of e-foldings. Superstring theory contains many axion-like particles (moduli fields) that can produce natural inflation. Current constraints on natural inflation models from three years of WMAP data are shown in figure 2 (from [10]). Natural inflation models with $f \gtrsim 0.7 M_{\mathrm{Pl}}$ and reasonable number of e-foldings fall within the $95 \%$ confidence level. Testable predictions of natural inflation that can be verified in upcoming cosmological observations [11]: for example, the existence of tensor modes, which although smaller than in other models should be detectable, and a very mild running of the spectral index $n_{s}$. With projected Planck error bars of order \pm 0.05 in $r$ and \pm 0.01 in $n_{s}$, the next generation of experiments should be able to check natural inflation.

Chain inflation [12] is a revamp of the old inflation idea [1] of a scalar field tunneling through an energy barrier to pass from a false to a true vacuum. In old inflation, the bubbles of true vacuum failed to percolate and thermalize in the exponentially expanding Universe, hence inflation would never end. In chain inflation, instead, a long sequence of catalyzed tunneling events is postulated, each event lasting only for a small number of e-foldings. It requires no fine-tuning of parameters, and it succeeds from the MeV to the GUT scale. Chain inflation is inspired by the large number of vacua in the string "landscape," which is the gazillion-minima potential of the multitude of moduli fields in superstring theory. In such models, the scalar fields are naturally coupled in a way that the slow-rolling of one field triggers the tunneling of another. The very small number of efoldings per tunneling event allows an efficient percolation and thermalization of the new-vacuum bubbles. While very interesting on account of its connection to superstring theory, chain inflation in the landscape awaits an observational test against the CMB and large-scale structure fluctuations because of the difficulty in computing the expected density fluctuations.

\section{Supersymmetric dark matter from moduli fields}

A completely different way of exploring the Universe before primordial nucleosynthesis is to search for particle relics that were produced at those early times and survive until the present. For example, particles 10 times heavier, or 1000 times heavier, than the proton, and having weak-force interaction strengths, could have been produced out of collisions in the primordial plasma during the first microsecond, or femtosecond, respectively. They would then have survived unscathed till the present time and would now account for the cold dark matter whose existence is inferred from a variety of cosmological measurements. These particles go under the name of weakly interacting massive particles, or WIMPs.

Dozens of experiments worldwide are searching for cold dark matter WIMPs. Despite several incongruous claims of having detected them, no experiment has uncontestedly supplied a positive signal.

The most studied candidate for the role of dark matter WIMP is a hypothetical particle called the neutralino (not to be confused with the neutrino). The neutralino is the supersymmetric partner of the electrically-neutral gauge and Higgs bosons. In most supersymmetric models it is the lightest stable supersymmetric particle.

\footnotetext{
${ }^{1}$ Moduli fields parametrize the size and shape of the compactified extra dimensions.
} 
Supersymmetry is a symmetry between particles of different spin. In theories with fundamental scalar fields, like the Standard Model, supersymmetry offers an ingenious way of keeping the scale of electroweak interactions much lower than the grand-unification scale or the Planck mass. It is also an indispensable ingredient in superstring theory, which seeks to unify general relativity and quantum mechanics. In a supersymmetric theory, every particle has a supersymmetric partner of the same mass and charge but of different spin. Since no particle with the mass and charge of the electron but different spin has ever been observed, supersymmetry must be realized as a broken symmetry, much as the electroweak symmetry in the Standard Model. Several mechanisms have been suggested for breaking supersymmetry, and each one of them leads to different predictions for the masses and couplings of elementary particles. The upcoming Large Hadron Collider at CERN will hopeful be able to produce supersymmetric particles for the first time.

The neutralino is a good cold dark matter candidate because (1) its relic density can be computed to high precision once the parameters of a supersymmetric model have been specified, and (2) model parameters can be chosen so that the neutralino density matches that of cold dark matter. Since the density of the latter has been recently measured with precision, the models selected by condition (2) lie on very thin slices of parameter space. Figure 3, from [14], displays one of these slices as the narrow line marked WMAP extending across a portion of the $m_{0}, m_{1 / 2}, \tan \beta, A_{0}$ parameter space of minimal supergravity (a particular pattern of supersymmetry breaking).

This scenario, however, may be completely different if the evolution of the Universe before nucleosynthesis does not follow the standard radiation-dominated paradigm [15]. To appreciate how the neutralino density is affected by changes in the early cosmology, it is helpful to review the standard evaluation of the neutralino density. At early times, neutralinos are produced in $\mathrm{e}^{+} \mathrm{e}^{-}, \mu^{+} \mu^{-}$, etc. collisions in the hot primordial soup (thermal production). Neutralino production ceases when the production rate becomes smaller than the Hubble expansion rate (a moment called freeze-out).

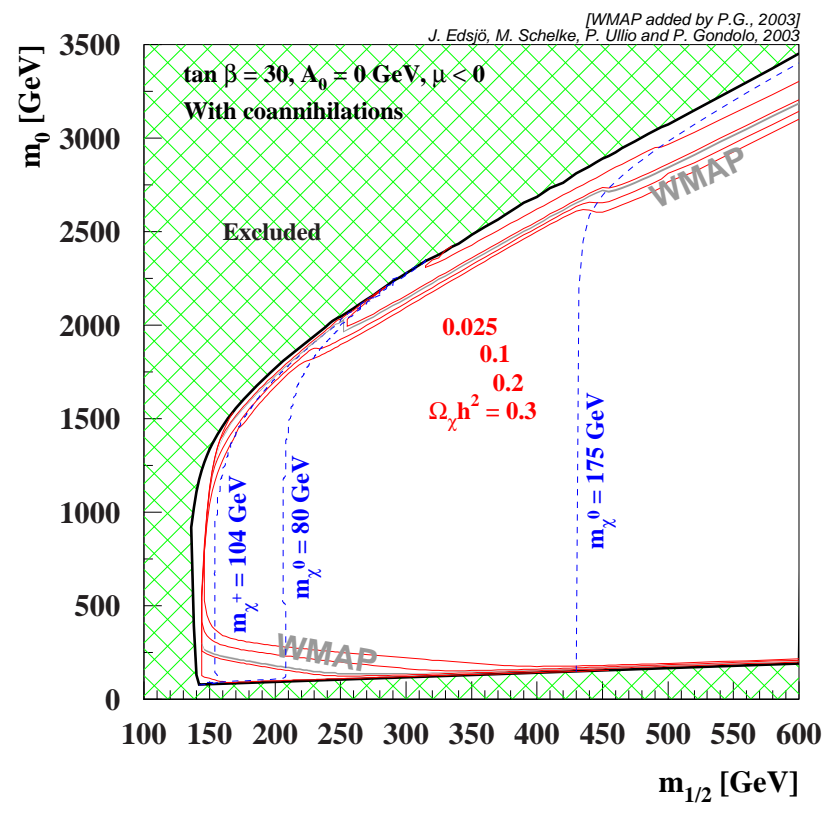

Figure 3: Narrow slices of minimal supergravity parameter space selected by the standard evaluation of the neutralino relic density (lines labeled WMAP). (Figure adapted from [14].) 
After freeze-out, the number of neutralinos per photon is constant. In this process, interactions before freeze-out keep neutralinos in chemical equilibrium with the soup, and the Boltzmann factor $e^{-m_{\chi} / T}$ lowers their number as the temperature decreases. The stronger the interactions, the later freeze-out occurs, and the fewer neutralinos are left. This leads to the inverse proportionality of relic density and annihilation cross section

$$
\Omega_{\chi} h^{2}=\frac{2 \times 10^{-10} \mathrm{GeV}^{-2}}{\langle\sigma v\rangle} .
$$

Here $\langle\sigma v\rangle$ is the thermal average of the annihilation cross section times $\chi \chi$ relative velocity.

In non-standard cosmologies, the neutralino density can be larger or smaller than its standard value. The density can decrease if photons are produced after freeze-out, since we can measure the number of neutralinos only relative to the number of photons (this effect is called entropy dilution). The density can instead increase if neutralinos are created out of chemical equilibrium from particle decays or topological defects (this is called non-thermal production). The density can also increase if the expansion rate at the moment of freeze-out is larger than its standard value (e.g. if the Universe is dominated not by radiation but by a scalar field like quintessence or a moduli field; in this case, the neutralino would freeze-out earlier, when it is more abundant).

A specific case of non-standard cosmology that may bear on superstring theory has been studied in $[16,17]$ : neutralino production from the decay of a scalar field $\phi$ while the Universe is dominated by the scalar field itself. This field can be a moduli field from superstring theory. In this case, the non-standard cosmology can be described by only two parameters: the reheating

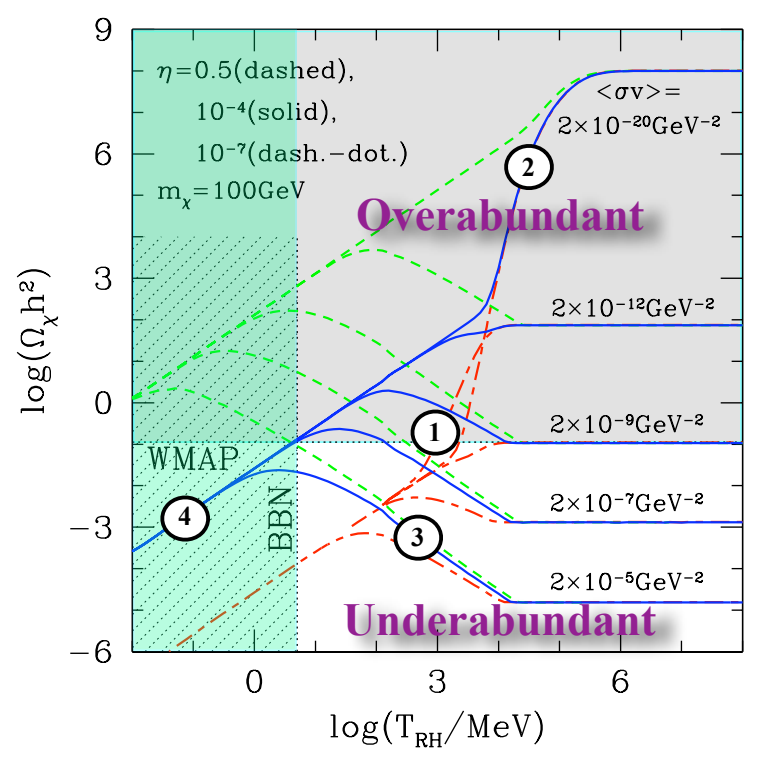

Figure 4: Neutralino density $\Omega_{\chi} h^{2}$ as a function of the reheating temperature $T_{\mathrm{RH}}$ for selected choice of the neutralino-neutralino annihilation cross section times relative velocity $\langle\sigma v\rangle$ and the number $\eta$ of neutralinos produced per $\phi$ decay. The neutralino mass is taken to be $100 \mathrm{GeV}$, corresponding to a freeze-out temperature of $\sim 10^{4} \mathrm{MeV}$. Big bang nucleosynthesis imposes $T_{\mathrm{RH}} \gtrsim 5 \mathrm{MeV}$. The observed cold dark matter density is indicated by the line labeled WMAP. (Figure adapted from [16].) 
temperature $T_{\mathrm{RH}}$, i.e. the temperature of the radiation when it becomes radiation dominated after the $\phi$ decays, and the ratio $b / m_{\phi}$ between the number of neutralinos produced per $\phi$ decay and the $\phi$ mass. Suitable combinations of these parameters can make the neutralino density equal to the cold dark matter density for almost any annihilation cross section. Figure 4 illustrates this point. In the figure, the neutralino density is plotted as a function of the reheating temperature for various annihilation cross sections and "branching ratios" $b$. On a given $\langle\sigma v\rangle$ line, the standard density corresponds to the constant segment at high reheating temperatures (on the right). At reheating temperatures lower than the standard freeze-out temperature (here $\sim 10^{4} \mathrm{MeV}$ ), the density can be decreased through entropy dilution along lines parallel to line 1 , if in chemical equilibrium, or line 2 , if out of chemical equilibrium. The density can also be increased through non-thermal production along lines parallel to line 3 , in chemical equilibrium, or line 4 , out of chemical equilibrium. Once a certain $\langle\sigma v\rangle$ is specified in a supersymmetric model, the neutralino relic density can be brought to that of cold dark matter (horizontal line labeled WMAP) by a suitable combination of thermal and non-thermal production, i.e. an appropriate choice of $T_{\mathrm{RH}}$ and $b / m_{\phi}$. This freedom disappears only for the highest cross sections (lowest standard densities), for which line 1 (equilibrium non-thermal production) may fail to intersect the WMAP line at $T_{\mathrm{RH}}$ greater than the BBN bound.

These considerations can be applied to the minimal supersymmetric standard model (the smallest supersymmetric model that contains the Standard Model and all possible soft-supersymmetry breaking terms). In this case, all but few models with $m_{\chi} \simeq m_{Z} / 2$ can have a neutralino relic density equal to that of cold dark matter on the condition that $m_{1 / 2}<m_{\phi}<m_{3 / 2}$, where $m_{3 / 2}$ is the gravitino mass, and that $b / m_{\phi}$ is related to $m_{\chi}$ and $T_{\mathrm{RH}}$ as in figure 5 .

The intriguing point is that these conditions are controlled by the physics at the grand-unified or Planck scale. The $m_{1 / 2}<m_{\phi}<m_{3 / 2}$ mass hierarchy depends on the hidden sector of the highenergy theory and on the kind of supersymmetry breaking. The value of $b$ depends on the choice of Kähler potential, superpotential, and gauge kinetic function. Ultimately, these parameters depend

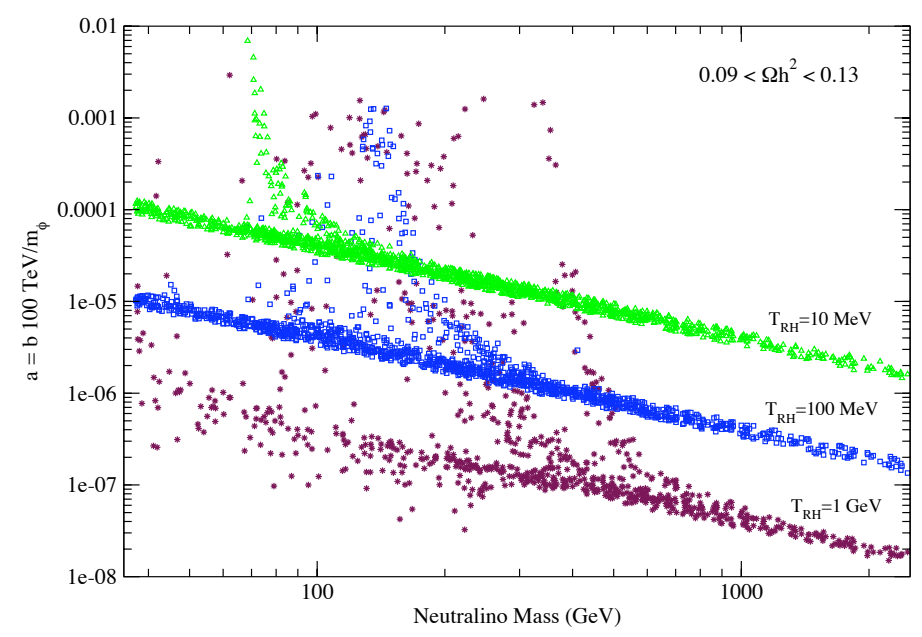

Figure 5: Cosmological constraints on the high-energy theory parameter $b / m_{\phi}$ as a function of the neutralino mass $m_{\chi}$ for a series of reheating temperatures $T_{\mathrm{RH}}$ (from [17]). 
on how the extra-dimensions are compactified, on the presence of string-theory fluxes, and on other details of the underlying fundamental theory. The strong cosmological constraints shown in figure 5 thus apply to the high-energy theory (superstring theory).

\section{Conclusions}

This presentation has concentrated on two ways to probe the physics of the Universe before nucleosynthesis: the analysis of $\mathrm{CMB}$ and large scale fluctuation data to reconstruct the inflaton potential, and the possible detection of supersymmetric dark matter particles to explore the highenergy theory at the Planck scale.

Cosmological observations are starting to probe inflation, but much work still remains to be done to go beyond the simplest models (e.g., an analysis that includes the full $k$-dependence, a calculation of the fluctuations in chain inflation, ...). Models like natural inflation and chain inflation can conceivably be implemented in superstring theories.

Neutralinos can be all of the cold dark matter almost anywhere in supersymmetric parameter space if one allows for non-thermal neutralino production form moduli field decays and low reheating temperatures.

An exciting situation would arise if dark matter searches would find neutralino dark matter and particle accelerators would discover supersymmetry where the standard neutralino density is too high. This would constitute indirect evidence of a non-standard cosmology before nucleosynthesis, and through the constraints on moduli fields delineated above may open an experimental window on superstring theory.

\section{References}

[1] A. H. Guth, Cosmological consequences of a first order phase transition in the SU(5) grand unified model, Phys. Rev. D 23 (1981) 347.

[2] S. Dodelson, W. H. Kinney, E. W. Kolb, Cosmic microwave background measurements can discriminate among inflation models, Phys. Rev. D 56 (1997) 3207 [astro-ph/9702166].

[3] E. W. Kolb, Dynamics of the inflationary era, 1999 [hep-ph/9910311].

[4] S. Dodelson, Modern Cosmology, Academic Press, San Diego, 2003.

[5] A. Riotto, Inflation and the theory of cosmological perturbations, lectures given at the Summer School on Astroparticle Physics and Cosmology, Trieste, 2002 [hep-ph/0210162].

[6] D. N. Spergel, et al. Wilkinson Microwave Anisotropy Probe (WMAP) three year results: implications for cosmology, 2006 [astro-ph / 0603449 ].

[7] H. V. Peiris, R. Easther, Recovering the inflationary potential and primordial power spectrum with a slow roll prior, 2006 [astro-ph/0603587].

[8] K. Freese, J. A. Frieman, A. V. Olinto, Natural inflation with pseudo Nambu-Goldstone bosons, Phys. Rev. Lett. 65 (1990) 3233.

[9] F. C. Adams, J. R. Bond, K. Freese, J. A. Frieman, A. V. Olinto, Natural inflation: particle physics models, power-law spectra for large-scale structure, and constraints from the Cosmic Background Explorer, Phys. Rev. D 47 (1993) 426. 
[10] W. H. Kinney, E. W. Kolb, A. Melchiorri, A. Riotto, private communication.

[11] K. Freese, W. H. Kinney, On natural inflation, Phys. Rev. D 70 (2004) 083512 [hep-ph/ 0404012 ].

[12] K. Freese, D. Spolyar, Chain inflation in the landscape: 'bubble bubble toil and trouble', JCAP 07 (2005) 007 [hep-ph/ 0412145$].$

[13] E. A. Baltz, private communication.

[14] J. Edsjö, M. Schelke, P. Ullio, P. Gondolo, Accurate relic densities with neutralino, chargino and sfermion coannihilations in mSUGRA, JCAP 04 (2003) 001 [hep-ph/ 030110 6].

[15] See, e.g., J. D. Barrow, Massive particles as a probe of the early Universe, Nucl. Phys. B208 (1982) 501; M. Kamionkowski, M. S. Turner, Thermal relics: do we know their abundance?, Phys. Rev. D 42 (1990) 3310; R. Jeannerot, X. Zhang, R. Brandenberger, Non-thermal production of dark matter from cosmic strings, JHEP 12 (1999) 003; D. J. H. Chung, E. W. Kolb, A. Riotto, Production of massive particles during reheating, Phys. Rev. D 60 (1999) 063504; T. Moroi, L. Randall, Wino cold dark matter from anomaly mediated SUSY breaking, Nucl. Phys. B 570 (2000) 455; P. Salati, Quintessence and the relic density of neutralinos, Phys. Lett. B571 (2003) 121.

[16] G. Gelmini, P. Gondolo, Neutralino with the right cold dark matter abundance in (almost) any supersymmetric model, 2006 [hep-ph/0602230].

[17] G. Gelmini, P. Gondolo, A. Soldatenko, C. Yaguna, The effect of a late decaying scalar on the neutralino relic density, 2006 [hep-ph/0605016]. 